\title{
Application of the RRSB, Pogosov, and Weining functions to describe the cumulative size characteristics of anthracites from the Krasnogorsky Mine
}

\author{
Vladimir Udovitsky*, Vladimir Kandinsky, Artem Begunov, and Natalia Mamontova \\ T.F. Gorbachev Kuzbass State Technical University, Department of Mineral Processing, \\ Vesennyaya st., 28, 650000 Kemerovo, Russian Federation
}

\begin{abstract}
Many researchers have found that the nature of the grain size distribution makes it possible to assume the regularity of such a distribution, despite the difference in the physical properties of minerals. There are quite a large number of approximating functions for describing the regularities of the separation of coal and mineral complexes by size, obtained experimentally. There is a need to develop a new methodological approach and tools for selecting the best approximation function. The paper presents the results of predicting the total size characteristics of anthracites of the Krasnogorsky mine by the RRSB, Pogosov, and Weining functions. The quality of the approximation is estimated by the value of the standard deviation. It is established that the Weining formula allows us to calculate with a minimum error the granulometric composition of anthracites of the named deposit in the range of fineness from 0 to 150 $\mathrm{mm}$.
\end{abstract}

\section{Introduction}

Studies of the size characteristics have shown that there is a certain regularity in the distribution of coal particles by size classes, for the description of which the functions discussed earlier can be used to predict the total size characteristics of the" plus " coal of the Kuznetsk basin in the size range from 0 to $300 \mathrm{~mm}$ [1].

The granulometric composition is performed by the sieve method. The method consists in sieving the fuel on sieves and determining the yields of the size classes. The size of the sides of the mesh cells of the sieves used in the sieve analysis is selected in accordance with Annex 1 of the state standard [2]. The main row includes sieves with the size of the cells $100,50,25,13,6,3,1,0.5 \mathrm{~mm}$.

At the enterprises of the fuel and energy complex, they do not always adhere to the main series of sieves, they perform a sieve analysis (to reduce the amount of work) according to the sizes of sieves holes that are traditionally accepted at this enterprise.

There is a need to determine the granulometric composition of different machine classes

*Corresponding author: $\underline{\text { uvi@kuzstu.ru }}$ 
in the preparatory and main processes, the values of the yields and ash content of which can be calculated by mathematical modeling methods based on experimental granulometric compositions.

To achieve this goal, the approximations of RRSB, A.M. Pogosov and A. Weining were used, which turned out to be the best at the stage of preliminary research of the distribution of coal particles by size classes [3].

In this paper, the tasks are set and solved:

- development of a new methodological approach for selecting the best approximation function when describing the total size characteristics using the example of anthracites from the Krasnogorsky mine, Mezhdurechensk;

- the function parameters are calculated using a problem-oriented software package using the least squares method. The quality of the approximations was estimated by the value of the standard deviation;

- the best approximation function is used to calculate the output values in the range from 0 to $150 \mathrm{~mm}$.

\section{Materials and Methods}

\subsection{Analytical expressions for describing the distribution of anthracite particles by size classes}

The team of authors of the monograph [4] notes that a significant event in the attempts to display the laws of particle size distribution using mathematical dependencies was the collective work presented on the one hand by P. Rozin and E. Rammler (1932) [5, 6], and on the other - by K. Sperling in collaboration with Mayer.

Studying the particle size distribution of coal dust, the authors concluded that it can be described using a formula that they characterized as a generalized exponential function. The dependence (in fractions of one) of the remainder $\mathrm{R}$ or sifting $\mathrm{D}$ of the crushed material on its fineness $x$ they presented as

$$
\begin{gathered}
R=e^{-b x^{n}}, \\
D=1-e^{-b x^{n}} .
\end{gathered}
$$

P. Rozin and E. Rammler showed the possibility of using functions (1) and (2) to describe the characteristics of the fineness of crushed materials used in the ceramic industry [7].

In 1936, J. Bennett [4], having accepted $b=\frac{1}{\frac{-n}{x}}$, presented functions (1) and (2) as

$$
\begin{gathered}
R=e^{-\left(\frac{x}{\bar{x}}\right)^{n},} \\
D=1-e^{-\left(\frac{x}{x}\right)^{n},}
\end{gathered}
$$

giving them a new interpretation. The parameter $\bar{x}$ is a particle size where the yield of all other particles whose size exceeds its value is $36.79 \%$. This is the equation in the entry of J. The Bannet equation, which is referred to in some countries as the Rosin-Rammler 
equation, in others as the RRS equation (the Rosin-Rammler-Sperling equation), or RRB or RRSB, has become widely used in world practice and is successfully used at the present time [8].

In this paper, the function (1) is written as

$$
y=\frac{a_{0} \sqrt{x}}{\exp \left(a_{1} / x\right)} .
$$

It is easy to see that after the linearization of function (5), the graphical representation of the total characteristic may well be a straight line.

The authors of $[4,9]$ indicate that the analysis of the accuracy of reflecting the actual distribution of coal by size classes using the RRSB equation, performed on the basis of processing data from sieve analyses of anthracites (more than 33 coal seams) and hard coals (more than 30 coal seams) In the Donetsk and Lviv-Volyn basins, he concluded that this formula either does not accurately describe the regularity of the distribution of coal size, or is applicable in a narrow range of size.

The publication [4] presents the results of the research of scientists who came to the conclusion that the weight distribution of the crushed material by size is consistent with the logarithmic-normal distribution law. However, the employees of Ukrniiugleobogaschenie, after conducting studies with coal and anthracite from 200 coal seams in the Donetsk and Lviv-Volyn basins, found that the total characteristics of most of the layers did not correspond to the logarithmic-normal distribution law.

N. K. Beloglazov [10] (1956) by converting the equation RRSB (5) obtained the function of the characteristic of the fineness of the products of grinding monomineral ores by "minus»

$$
y=200 a_{0} x^{a_{1}} /\left(1+a_{0} x^{a_{1}}\right) .
$$

Experimental verification conducted by the B.A. Zemskova [5] showed that the equation N.K. Beloglazova the best agreement with the experimental data for particles of small and medium size and a wider range than formula RRSB.

A.M. Pogosov [5, 11] (1960) tried to extend the range of the actual grain size was described by the equation Beloglazova (6), by replacing factor "200" to "100". The formula he proposed has the form

$$
y=100 a_{0} x^{a_{1}} /\left(1+a_{0} x^{a_{1}}\right) .
$$

As the further verification of the function (7) carried out by the authors of this work will show, the formula of A.M. Pogosov surpasses the RRSB function in terms of the reliability of the description of the total characteristics of the fineness of the hard coals of the Kuznetsk basin in the range of [0-100] $\mathrm{mm}$.

The study of the characteristics of the fineness of the grinding products and deep theoretical research allowed A. Weining to present the grinding process, which cannot be described using two-parameter equations. He comes to the conclusion that "in general, the curve of the numerical distribution of the particles of the grinding products should be described by the equation of a high-degree parabola" [4]

$$
n=A+B x+C x^{2}+D x^{3} \text {. }
$$

A. Weining considers this function as an approximate one, which equalizes the experimental particle size distribution quite well.

The authors of the monograph [4] come to the conclusion that A. Weining proposes the formula (8), "based on speculative assumptions, and gets a complex, inconvenient equation for solving specific problems, which he himself suggests replacing with a simpler one."

Since this function will be used in our research, we will present it in the form of formula (9) and call it the A. Weining function 


$$
y=a_{0}+a_{1} x+a_{2} x^{2}+a_{3} x^{3} .
$$

As further computational experiments with third-degree polynomials will show when describing the total characteristics of the black coal of the Kuznetsk basin, A. Weining holds the primacy in the choice of approximations of the third degree, and the quality of the approximation (9) exceeded all the functions of the first degree considered above when describing the total characteristics of the fineness of the "plus".

\subsection{Mathematical model}

The parameters of the functions were calculated by a problem-oriented software package using the well-known least squares method. The peculiarity of using the method is that the functions must be linear or reduced to a linear form.

The sequence of mathematical transformations for obtaining systems of linear algebraic equations (SLAE) is shown below.

$$
y=100 / \exp \left(a_{0} \cdot x^{a 1}\right)-\text { the transformed equation } \operatorname{RRSB}(5)
$$

We reduce the function to a linear form

$$
\begin{gathered}
100 / y=\exp \left(a_{0} \cdot x^{a 1}\right), \\
\ln (\ln (100 / y))=\ln a_{0}+a_{1} \cdot \ln x, \\
c=\ln a_{0},
\end{gathered}
$$

Let's select the parameters of the function $\left(a_{0}\right.$ and $\left.a_{1}\right)$ so as to provide a minimum of the sum of the squares of deviations between the experimental values of the function and the calculated ones

$$
U K\left(c, a_{1}\right)=1 / n \Sigma\left(c+a_{1} \cdot \ln \left(x_{i}\right)-\ln \left(\ln \left(100 / y_{i}\right)\right)\right)^{2}
$$

Differentiating by $\mathrm{a}_{0}$ and $\mathrm{a}_{1}$

$$
\begin{gathered}
\partial U K / \partial c=2 \cdot 1 / n \Sigma\left(c+a_{1} \cdot \ln \left(x_{i}\right)-\ln \left(\ln \left(100 / y_{i}\right)\right)\right) \cdot 1=0 \\
\partial U K / \partial a_{1}=2 \cdot 1 / n \Sigma\left(c+a_{1} \cdot \ln \left(x_{i}\right)-\ln \left(\ln \left(100 / y_{i}\right)\right)\right) \cdot \ln \left(x_{i}\right)=0
\end{gathered}
$$

getting the system

$$
\begin{gathered}
c+a_{1} \cdot 1 / n \Sigma \ln \left(x_{i}\right)=1 / n \Sigma \ln \left(\ln \left(100 / y_{i}\right)\right) \\
c \cdot 1 / n \Sigma \ln x_{i}+a_{1} \cdot 1 / n \Sigma \ln ^{2}\left(x_{i}\right)=1 / n \Sigma \ln \left(x_{i}\right) \cdot \ln \left(\ln \left(100 / y_{i}\right)\right)
\end{gathered}
$$

Let's introduce the notation

$$
\begin{gathered}
S_{0}=1, S_{1}=1 / n \Sigma \ln \left(x_{i}\right), S_{2}=1 / n \sum \ln ^{2}\left(x_{i}\right) \\
B_{0}=1 / n \sum \ln \left(\ln \left(100 / y_{i}\right)\right), B_{1}=1 / n \sum \ln \left(x_{i}\right) \cdot \ln \left(\ln \left(100 / y_{i}\right)\right)
\end{gathered}
$$

Let's write the system in a "new" form

$$
\begin{aligned}
& c \cdot S_{0}+a_{1} \cdot S_{1}=B_{0} \\
& c \cdot S_{1}+a_{1} \cdot S_{2}=B_{1}
\end{aligned}
$$

Having solved the SLAE, we define the parameter $a_{0}$ 


$$
a_{0}=\exp (c)
$$

$y=\frac{100 a_{0} x^{a 1}}{\left(1+a_{0} x^{a 1}\right)}-$ the transformed formula of A.M. Pogosov (7)

$$
\begin{gathered}
\ln (y /(100-y))=\ln \left(a_{0}\right)+a_{1} \ln (x) \\
c=\ln \left(a_{0}\right)
\end{gathered}
$$

$$
\begin{gathered}
U K\left(c, a_{1}\right)=1 / n \Sigma\left(c+a_{1} \ln \left(x_{i}\right)-\ln \left(y_{i} /\left(100-y_{i}\right)\right)\right)^{2} \\
\partial U K / \partial c=2 \cdot 1 / n \Sigma\left(c+a_{1} \ln \left(x_{i}\right)-\ln \left(y_{i} /\left(100-y_{i}\right)\right)\right) \cdot 1=0 \\
\partial U K / \partial a_{1}=2 \cdot 1 / n \Sigma\left(c+a_{1} \ln \left(x_{i}\right)-\ln \left(y_{i} /\left(100-y_{i}\right)\right)\right) \cdot \ln \left(x_{i}\right)=0 \\
c+a_{1} 1 / n \Sigma \ln \left(x_{i}\right)=1 / n \Sigma\left(\ln \left(y_{i} /\left(100-y_{i}\right)\right)=0\right. \\
c 1 / n \Sigma \ln \left(x_{i}\right)+a_{1} 1 / n \Sigma \ln ^{2}\left(x_{i}\right)=1 / n \Sigma \ln \left(x_{i}\right) \cdot \ln \left(y_{i} /\left(100-y_{i}\right)\right)=0 \\
S_{0}=1, S_{1}=1 / n \Sigma \ln \left(x_{i}\right), S_{2}=1 / n \Sigma \ln ^{2}\left(x_{i}\right) \\
B_{0}=1 / n \Sigma \ln \left(y_{i} /\left(100-y_{i}\right)\right), B_{1}=1 / n \Sigma \ln \left(x_{i}\right) \cdot \ln \left(y_{i} /\left(100-y_{i}\right)\right) \\
c S_{0}+a_{1} S_{1}=B_{0} \\
c S_{1}+a_{1} S_{2}=B_{1} \\
a_{0}=\exp (c)
\end{gathered}
$$

$y=a_{0}+a_{1} x+a_{2} x^{2}+a_{3} x^{3}-$ the Weining formula (8)

$$
\begin{gathered}
U K\left(a_{0}, a_{1}, a_{2}, a_{3}\right)=1 / \mathrm{n} \Sigma\left(a_{0}+a_{1} x_{\mathrm{i}}+a_{2} x^{2}{ }_{\mathrm{i}}+a_{3} x^{3}{ }_{\mathrm{i}}-y_{\mathrm{i}}\right)^{2} \\
\partial U K / \partial a_{0}=2 \cdot 1 / \mathrm{n} \Sigma\left(a_{0}+a_{1} x_{i}+a_{2} x^{2}{ }_{i}+a_{3} x^{3}{ }_{i}-y_{i}\right) 1=0 ; \\
\partial U K / \partial a_{1}=2 \cdot 1 / \mathrm{n} \Sigma\left(a_{0}+a_{1} x_{i}+a_{2} x^{2}{ }_{i}+a_{3} x^{3}{ }_{i}-y_{i}\right) x_{i}=0 ; \\
\partial U K / \partial a_{2}=2 \cdot 1 / \mathrm{n} \Sigma\left(a_{0}+a_{1} x_{i}+a_{2} x^{2} \mathrm{i}+a_{3} x^{3}{ }_{i}-y_{i}\right) x^{2}=0 ; \\
\partial U K / \partial a_{3}=2 \cdot 1 / \mathrm{n} \Sigma\left(a_{0}+a_{1} x_{i}+a_{2} x^{2}{ }_{i}+a_{3} x^{3}{ }_{i}-y_{i}\right) x_{i}^{3}=0 ;
\end{gathered}
$$

$$
a_{0}+a_{1} 1 / \mathrm{n} \sum x_{i}+a_{2} 1 / \mathrm{n} \sum x^{2}{ }_{i}+a_{3} 1 / n \sum x_{i}{ }_{i}=1 / \mathrm{n} \Sigma y_{i} ;
$$

$a_{0} 1 / \mathrm{n} \sum x_{i}+a_{1} 1 / \mathrm{n} \Sigma x^{2}{ }_{i}+a_{2} 1 / \mathrm{n} \sum x_{i}^{3}+a_{3} 1 / \mathrm{n} \Sigma x^{4}{ }_{i}=1 / \mathrm{n} \sum x_{i} y_{i} ;$ $a_{0} 1 / \mathrm{n} \sum x^{2}{ }_{i}+a_{1} 1 / \mathrm{n} \sum x^{3}{ }_{i}+a_{2} 1 / \mathrm{n} \sum x^{4}{ }_{i}+a_{3} 1 / \mathrm{n} \sum x^{5}{ }_{i}=1 / \mathrm{n} \Sigma x^{2}{ }_{i} y_{i} ;$ $a_{0} 1 / \mathrm{n} \sum x_{i}^{3}+a_{1} 1 / \mathrm{n} \sum x_{i}^{4}+a_{2} 1 / \mathrm{n} \sum x_{i}^{5}+a_{3} 1 / \mathrm{n} \sum x_{i}^{6}=1 / \mathrm{n} \sum x^{3}{ }_{i} y_{i} ;$

$$
\begin{gathered}
S_{0}=1 ; S_{1}=1 / \mathrm{n} \sum x_{i} ; S_{1}=1 / \mathrm{n} \sum x_{i}^{2} ; S_{3}=1 / \mathrm{n} \sum x_{i}^{3} \\
S_{4}=1 / \mathrm{n} \sum x^{4}{ }_{i} ; S_{5}=1 / \mathrm{n} \sum x^{5} ; S_{6}=1 / \mathrm{n} \sum x_{i}^{6} ; \\
B_{0}=1 / \mathrm{n} \Sigma y_{i} ; B_{1}=1 / \mathrm{n} \sum y_{i} x_{i} ; B_{2}=1 / \mathrm{n} \sum y_{i} x^{2}{ }_{i} ; B_{3}=1 / \mathrm{n} \Sigma y_{i} x^{3}{ }_{i},
\end{gathered}
$$




$$
\begin{array}{lllll}
a_{0} & S_{0}+a_{1} & S_{1}+a_{2} & S_{2}+a_{3} & S_{3}=B_{0} \\
a_{0} & S_{1}+a_{1} & S_{2}+a_{2} & S_{3}+a_{3} & S_{4}=B_{1} \\
a_{0} & S_{2}+a_{1} & S_{3}+a_{2} & S_{4}+a_{3} & S_{5}=B_{2} \\
a_{0} & S_{3}+a_{1} & S_{4}+a_{2} & S_{5}+a_{3} & S_{6}=B_{3} .
\end{array}
$$

Thus, the problem of determining the parameters of the approximating functions (5), (7) and (8) was reduced to solving systems of linear algebraic equations.

After calculating the values of $S_{\mathrm{i}}$ and $B_{\mathrm{i}}$ from the presented systems of linear algebraic equations, the parameters of $a_{\mathrm{i}}$ were determined by the least squares method.

To find the parameters from the system of linear algebraic equations, we used:

- in Excel, the Kramer method is the function MOPRED (array), which returns the determinant of the matrix;

- in TurboPascal - the Gaussian method (reducing the matrix to a triangular form).

Assumptions made in the calculations:

1. The size of the sieve holes, equal to $100 \mathrm{~mm}$, was taken as 99.99999 ;

2. The total output of classes equal to $100 \%$ was assumed to be 99.99999 ;

3. The particle size larger than "zero" was calculated as $>0.00001 \mathrm{~mm}$.

The quality of the approximations was estimated by the value of the standard deviation, Sigma, represented between the real and calculated values of the total outputs of the size classes (the last rows of Tables 1-4)

\section{Results and Discussion}

3.1 Sample 1. Ordinary coal of the A brand, plast XXXI (uch. No. 4), for battle ex. No. 457, maximum size $150 \mathrm{~mm}$

Table 1. Results of calculation of the granulometric composition of sample 1.

\begin{tabular}{|c|c|c|c|c|}
\hline \multirow{2}{*}{ Class, $m m$} & \multicolumn{4}{|c|}{ Output of classes by "plus", \% } \\
\cline { 2 - 5 } & \multirow{2}{*}{ real } & \multicolumn{3}{|c|}{ calculated by function } \\
\cline { 2 - 5 } & 21,0 & RRSB & Pogosova & Weining \\
\hline $70-150$ & 9.2 & 21.7 & 21.0 \\
\hline $40-70$ & 39,1 & 26.5 & 33.7 & 39.5 \\
\hline $25-40$ & 56,2 & 44.4 & 45.8 & 55.6 \\
\hline $13-25$ & 73,3 & 66.5 & 63.2 & 73.0 \\
\hline $2-13$ & 89,9 & 94.4 & 92.9 & 92.9 \\
\hline $0,5-2$ & 95,5 & 98.7 & 98.3 & 96.0 \\
\hline $0-0,5$ & 100,0 & 100.0 & 100.0 & 97.0 \\
\hline Sigma & & 8.57 & 6.06 & 1.64 \\
\hline
\end{tabular}

3.2 Sample 2. Ordinary coal of the A brand, plast XXXI (uch. No. 3), for battle ex. No. 344, maximum size $150 \mathrm{~mm}$ 
Table 2. Results of the calculation of the granulometric composition of sample 2.

\begin{tabular}{|c|c|c|c|c|}
\hline \multirow{3}{*}{ Class, mm } & \multicolumn{4}{|c|}{ Output of classes by "plus", \% } \\
\cline { 2 - 5 } & \multirow{2}{*}{ real } & \multicolumn{3}{|c|}{ calculated by function } \\
\cline { 2 - 5 } & & $R R S B$ & Pogosova & Weining \\
\hline $70-150$ & 3,2 & 8.7 & 16.6 & 3.0 \\
\hline $40-70$ & 29,8 & 26.2 & 27.3 & 31.7 \\
\hline $25-40$ & 63,1 & 44.4 & 39.0 & 58.9 \\
\hline $13-25$ & 77,4 & 66.8 & 57.3 & 80.0 \\
\hline $2-13$ & 91,4 & 94.7 & 91.8 & 95.2 \\
\hline $0,5-2$ & 98,1 & 98.8 & 98.2 & 96.8 \\
\hline $0-0,5$ & 100,0 & 100.0 & 100.0 & 97.3 \\
\hline Sigma & & 8.58 & 12.9 & 2.71 \\
\hline
\end{tabular}

3.3 Sample 3. Ordinary coal of the A brand, plast XXXII-XXXIII (uch. No. 4), for battle ex. No. 457, maximum size $150 \mathrm{~mm}$

Table 3. Results of calculation of the granulometric composition of sample 3

\begin{tabular}{|c|c|c|c|c|}
\hline \multirow{2}{*}{ Class, $m m$} & \multicolumn{4}{|c|}{ Output of classes by "plus", \% } \\
\cline { 2 - 5 } & \multirow{2}{*}{ real } & \multicolumn{3}{|c|}{ calculated by function } \\
\cline { 2 - 5 } & 20,6 & 18.5 & 29.6 & 20.3 \\
\hline $100-150$ & 37,0 & 31.0 & 38.0 & 38.1 \\
\hline $70-150$ & 56,7 & 51.8 & 52.6 & 55.0 \\
\hline $40-70$ & 68,0 & 66.0 & 64.7 & 67.3 \\
\hline $25-40$ & 78,8 & 81.2 & 78.5 & 80.5 \\
\hline $13-25$ & 88,0 & 91.0 & 89.3 & 90.0 \\
\hline $6-13$ & 94,3 & 95.5 & 94.5 & 94.6 \\
\hline $3-6$ & 99,1 & 98.5 & 98.2 & 97.7 \\
\hline $1-3$ & 99,6 & 99.7 & 99.7 & 99.1 \\
\hline $0.2-0$ & 100,0 & 100.0 & 100.0 & 99.4 \\
\hline $0-0.2$ & & 2.88 & 3.35 & 1.21 \\
\hline Sigma & & & & \\
\hline
\end{tabular}

3.4 Sample 4. Ordinary coal of the A brand, XXXI (uch. No. 4), warehouse No. 3, maximum size $150 \mathrm{~mm}$

Table 4. Results of calculation of the granulometric composition of sample 4

\begin{tabular}{|c|c|c|c|c|}
\hline \multirow{3}{*}{ Class, $m m$} & \multicolumn{4}{|c|}{ Output of classes by "plus", \% } \\
\cline { 2 - 5 } & \multirow{2}{*}{ real } & \multicolumn{3}{|c|}{ calculated by function } \\
\cline { 2 - 5 } & & RRSB & Pogosova & Weining \\
\hline $70-150$ & 22,0 & 7.9 & 21.0 & 21.8 \\
\hline $40-70$ & 34,2 & 24.6 & 32.9 & 35.8 \\
\hline $25-40$ & 54,5 & 42.7 & 45.1 & 51.5 \\
\hline $13-25$ & 69,5 & 65.3 & 62.7 & 69.6 \\
\hline $6-13$ & 80,7 & 82.9 & 79.6 & 82.8 \\
\hline $3-6$ & 86,3 & 91.4 & 89.3 & 89.1 \\
\hline $1-3$ & 92,7 & 97.2 & 96.5 & 93.5 \\
\hline $0-1$ & 100,0 & 100.0 & 100.0 & 95.8 \\
\hline Sigma & & 7.91 & 4.51 & 2.30 \\
\hline
\end{tabular}


Computational experiments have established:

- when describing the total characteristics of the "plus" anthracites of the Krasnogorsky mine in the particle size range from 0 to $150 \mathrm{~mm}$, the Weining function (8) was the best in all four cases;

* the principle of the methodological approach, shown in [1], on the feasibility of using problem-oriented software systems that include several approximations, from which the best one is selected by the smallest value of the standard deviation, is confirmed.

\section{References}

1. V. Udovitsky, V. Kandinsky. Proceedings of the 9th China-Russia Symposium «Coal in the 21st Century: Mining, Intelligent Equipment and Environment Protection», (2018)

2. GOST 2093-82. The fuel is solid. The sieve method for determining the granulometric composition, (1983)

3. V. Kandinsky Proceedings of the XX International Symposium named after Academician M. A. Usov of students and young scientists, dedicated to the 120th anniversary of the founding of the Tomsk Polytechnic University. Volume 2, (2016)

4. A.M. Kotkin, M.N. Yampolsky, K.D. Gerashchenko. Evaluation of coal enrichment capacity and efficiency of enrichment processes, (Nedra, Moscow, 1982)

5. B.A. Zemlyakov. Forecasting the characteristics of coal enrichment, (Nedra, Moscow, 1978)

6. S.E. Andreev, V.V. Tovarov, V.A. Perov. Regularities of grinding and calculation of characteristics of granulometric composition, (Metallurgizdat, Moscow, 1959).

7. P. Rosin, E. Rammler. Berichte der keramischen Gesellshaft, (1934)

8. P. Gao, T.S. Zhang, J.X. Wei, Q.J. Yu. Evaluation of RRSB distribution and lognormal distribution for describing the particle size distribution of graded cementitious materials. Powder Technology, 331, (2018)

9. A.M. Khesin, V.E. Fedorchenko, M.N. Yampolsky. Technique and technology of coal enrichment. Vol. IV, (1965)

10. N.K. Beloglazov. Zapiski LGI, (1956)

11. A.M. Pogosov. Mining, processing and metallurgy of non-ferrous metals, (1960) 